\title{
]jfis
}

\section{Fuzzy Regression Model Using Trapezoidal Fuzzy Numbers for Re-auction Data}

\author{
II Kyu Kim ${ }^{1}$, Woo-Joo Lee ${ }^{2}$, Jin Hee Yoon ${ }^{3}$, and Seung Hoe Choi ${ }^{4}$ \\ ${ }^{1}$ Department of Real Estate and Finance, Gwangju University, Gwangju, Korea \\ ${ }^{2}$ Department of Mathematics, Yonsei University, Seoul, Korea \\ ${ }^{3}$ School of Mathematics and Statistics, Sejong University, Seoul, Korea \\ ${ }^{4}$ School of Liberal Arts and Science, Korea Aerospace University, Goyang, Korea
}

\begin{abstract}
Re-auction happens when a bid winner defaults on the payment without making second in-line purchase declaration even after determining sales permission. This is a process of selling under the court's authority. Re-auctioning contract price of real estate is largely influenced by the real estate business, real estate value, and the number of bidders. This paper is designed to establish a statistical model that deals with the number of bidders participating especially in apartment re-auctioning. For these, diverse factors are taken into consideration, including ratio of minimum sales value from the point of selling to re-auctioning, number of bidders at the time of selling, investment value of the real estate, and so forth. As an attempt to consider ambiguous and vague factors, this paper presents a comparatively vague concept of real estate and bidders as trapezoid fuzzy number. Two different methods based on the least squares estimation are applied to fuzzy regression model in this paper. The first method is the estimating method applying substitution after obtaining the estimators of regression coefficients, and the other method is to estimate directly from the estimating procedure without substitution. These methods are provided in application for re-auction data, and appropriate performance measure is also provided to compare the accuracies.
\end{abstract}

Keywords: Re-auction, Trapezoidal fuzzy number, Fuzzy regression model, Least squares estimation

\section{Introduction}

Received: Jan. 5, 2016

Revised : Mar. 22, 2016

Accepted: Mar. 24, 2016

Correspondence to: Seung Hoe Choi (shchoi@kau.ac.kr)

(T)The Korean Institute of Intelligent Systems

(c)This is an Open Access article distributed under the terms of the Creative Commons Attribution Non-Commercial License (http://creativecommons.org/licenses/ by-nc/3.0// which permits unrestricted noncommercial use, distribution, and reproduction in any medium, provided the original work is properly cited.
There are two types of real estate auction. The one is a compulsory auction which is requested by the creditor when the real estate is owned by the debtor. The other is a voluntary auction requested by a holder of a real right granted by way of security. Re-auction happens when a successful bidder has not paid the balance until payment due without second in-line purchase declaration even after determining sales permission. It is a process of selling under the court's authority. Reasons for defaulting on the payment can involve legal problems or economic problems. Legal reasons may be caused by analyzing the real estate title, assessing market price, and granting loans. Especially when the real estate market is in decline, re-auction is likely done because of the default on the payment of purchasing the deal at a higher price than the market price rather than because of the flaw of real estate or mistake of the analysis of real estate title. Thus, a bidding pricing is very important in real estate auction [1-3].

However, many bidders often ruin the investment estimate the bidding price simply since 
they based on their past experiences, intuitions, and market price. Dealing the real estate auction does not occur under normal condition: it occurs under a special circumstance where a bidder assesses the bidding price [3, 4]. As a result, price of the winning bid decreases as the number of bidders increases, and vice versa. Thus, in such type of deals, estimating the number of bidders is extremely significant.

Study on the real estate auction has been conducted in various fields, which may range from examining problems of auctioning process, analyzing the legal title, to discovering different types of case studies. Nevertheless, there has not been much study neither on the bidding price nor on the degree of bidding which chiefly affects the bidding price. One of the major problems of establishing a mathematical model of the real estate auction is the problem of uncertainty [2, 4]. There are two types of uncertainties: stochastic uncertainty whose uncertainty can be naturally resolved as time passes or as the experiment proceeds, and fuzzy uncertainty whose uncertainty is caused by an unclear distinction between different groups.

It seems more realistic to literally if not qualitatively express the investment value of real estate, which is deemed to influence the real estate auction, rather than presenting them as real numbers with definitive values. Hence, Zadeh [5, 6] suggested fuzzy theory in order to express literal variables in mathematical form. More specifically, he established the fuzzy control theory to explain fuzzy uncertainty in terms of ambiguity and vagueness, and to establish a necessary system for handling information expressed in such ambiguous or vague manners. Fuzzy theory quantifies ambiguity and vagueness by applying the degree of fuzziness and fuzzy measure. It also handles inference, evaluation, decision making based on quantified data.

This paper considers the ratio of minimum sales value from the point of selling to the point of re-auctioning, number of bidders at the time of selling, and investment value of the real estate as discrimination variable in order to construct a statistical model of the number of bidders participating in re-auctioning. Since investment value of the real estate and the number of bidders are ambiguous and vague in this particular suggested model, trapezoid fuzzy number is used as a statistical variable. As well, this paper uses the least square method in order to estimate the regression model in accordance with the number of bidders, using the trapezoid fuzzy number.

Also, two different methods based on the least squares estimations are applied to fuzzy regression model. The first method is to find the estimated values applying substitution after obtaining the estimators of regression coefficients, and the second method is to find the estimated values directly from the estimating procedure without substitution. These methods are provided in application for re-auction data, and proper performance measure is also provided to compare the accuracies in Section 2.

\section{Fuzzy Regression Model Using Trapezoidal Fuzzy Numbers}

Fuzzy regression analysis was first introduced by Tanaka and Hayashi [7] and Tanaka et al. [8] in order to apply linguistic or vague data to regression analysis. Fuzzy regression analysis using triangular fuzzy numbers and trapezoidal fuzzy number have been studied in many works [9-15]. The theoretical studeis regarding fuzzy regression model have been investigated in [16-18].

A fuzzy number can be expressed differently due to subjective points of view while a crisp number is expressed uniquely. However, expression of a fuzzy number is based on the objective observation, therefore a fuzzy number includes subjective concept as well as objective concept. Triangular fuzzy numbers and trapezoidal fuzzy numbers have been widely used to express these two different kinds of concepts. To analyze re-auction data, we introduce a fuzzy regression model with trapezoidal fuzzy input and outputs as follows:

$$
Y\left(X_{i}\right)=A_{0} \oplus A_{1} \otimes X_{i 1} \oplus \cdots \oplus A_{p} \otimes X_{i p} \oplus E_{i},
$$

where $X_{i j}$ is the $j$-th observation of $i$-th explanatory variable, $A_{i}$ is the fuzzy regression coefficient, and $Y\left(X_{i}\right)$ is the response variable. $\oplus$ and $\otimes$ is the addition and multiplication of fuzzy numbers, respectively. For the arithmetic operations, see [19] 20|. The membership function $M_{A}(\cdot)$ of a trapezoidal fuzzy number $(\mathrm{TrFN}) A=\left(l_{a}^{e}, l_{a}^{p}, r_{a}^{p}, r_{a}^{e}\right)$ can be expressed as follows:

$$
\mu_{A}(x)=\left\{\begin{array}{ccc}
0 & \text { if } \quad x<l_{a}^{e} \\
\frac{x-l_{a}^{e}}{l_{a}^{p}-l_{a}^{e}} & \text { if } \quad l_{a}^{e}<x<l_{a}^{p} \\
1 & \text { if } \quad l_{a}^{p}<x<r_{a}^{p} \\
\frac{r_{a}^{e}-x}{r_{a}^{e}-r_{a}^{p}} & \text { if } \quad r_{a}^{e}<x<r_{a}^{p} \\
0 & \text { if } \quad x>r_{a}^{e},
\end{array}\right.
$$

where $l_{a}^{e}$ and $r_{a}^{e}$ are two end points [13, 21], $l_{a}^{p}$ and $r_{a}^{p}$ and are two peak points (see Figure 1 ).

If $r_{a}^{e}-r_{a}^{p}=l_{a}^{p}-l_{a}^{e}$, the trapezoidal fuzzy number $A$ is called a symmetric trapezoidal fuzzy number (symmetric $\operatorname{TrFN}$ ). If the 


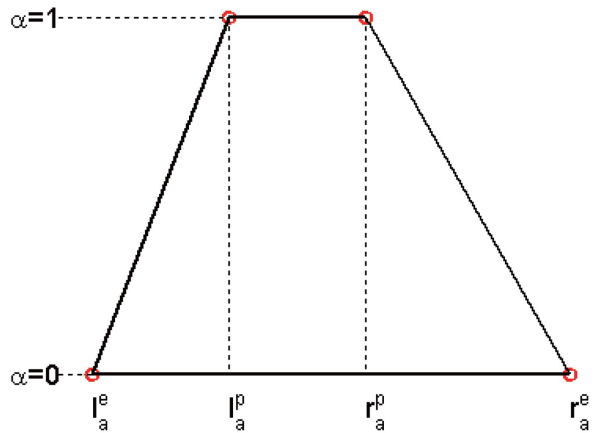

Figure 1. Trapezoidal fuzzy number $A=\left(l_{a}^{e}, l_{a}^{p}, r_{a}^{p}, r_{a}^{e}\right)$.

left endpoint $l_{a}^{e}$ is a positive number, then $A$ is called a positive TrFN. From practical point of view, note that the independent variables which are assigned with only positive or nonnegative fuzzy numbers, have been frequently used in several studies [13, 22, 24]. And if $l_{a}^{p}=r_{a}^{p}=p_{a}, A=\left(l_{a}^{e}, p_{a}, r_{a}^{e}\right)$ is a trangular fuzzy number (TFN) [7, 10, 11, 25, 26].

The support of a fuzzy set is defined by $S(A)=\{x \in$ $\left.R \mid M_{A}(x)>0\right\}=\left(l_{A}(0), r_{A}(0)\right)$. For any $\alpha$ in $[0,1]$, the $\alpha-$ level set is defined by $A(\alpha)=\left\{x \in R \mid M_{A}(x) \geq \alpha\right\}$, and $\alpha$-level set can be expressed by $A(\alpha)=\left[l_{A}(\alpha), r_{A}(\alpha)\right]$, where $l_{A}(\alpha)=l_{a}^{e}+\left(l_{a}^{p}-l_{a}^{e}\right) \alpha$ and $r_{A}(\alpha)=r_{a}^{e}+\left(r_{a}^{p}-r_{a}^{e}\right) \alpha$. From the model (1) with positive TrFN as input and output, $\alpha$-level set of $Y\left(X_{i}\right)$ with trapezoidal fuzzy inputs $X_{i}=\left[X_{i 1}, X_{i 2}, \cdots, X_{i p}\right]$ can be expressed by $Y\left(X_{i}\right)(\alpha)=\left[l_{Y\left(X_{i}\right)}(\alpha), r_{Y\left(X_{i}\right)}(\alpha)\right]$, where

$$
l_{Y\left(X_{i}\right)}(a)=\sum_{k=0}^{p} l_{A_{k}}(\alpha) \cdot l_{X_{i k}}(\alpha)+l_{E_{i}}(\alpha)
$$

and

$$
r_{Y\left(X_{i}\right)}(a)=\sum_{k=0}^{p} r_{A_{k}}(\alpha) \cdot r_{X_{i k}}(\alpha)+r_{E_{i}}(\alpha)
$$

Zadeh [6] suggested the extension principle to define the membership functions of a function of fuzzy numbers. Operations between fuzzy numbers can be defined based on the extension principle. Further he suggested the resolution identity. A fuzzy number can be expressed by $\alpha$-level set based on the resolution identity. Therefore, $Y\left(X_{i}\right)$ can be estimated based on its $\alpha$ - level set $Y\left(X_{i}\right)(\alpha)$ after estimating the regression coefficients $A_{k}(k=0, \cdots, p)$. The fuzzy regression coefficient $A_{k}$ can be estimated as follows:

In case the membership function of regression coefficient $A_{k}$ is known, $A_{k}$ can be estimated by estimating the parameters of membership function of $A_{k}$ using specific estimated $\alpha$-level set
$A_{k}(\alpha)$

However, if the membership function of $A_{k}$ is unknown, it should be estimated applying non-parametric method to estimated alpha-level sets of the regression coefficients after appropriate number of alpha-level sets are properly calculated. In case the membership function of regression coefficient $A_{K}$ is unknown, $A_{k}$ can be estimated by estimating finite number of $\alpha$-level sets of $A_{k}$ and applying proper estimation method for its membership function.

Next, the estimation procedure for the regression coefficients is proposed when the membership functions of those are known. In order to estimate the regression coefficients of (1), the regression model using trapezoidal fuzzy numbers for re-auction, 5 steps are proposed as follows: we suggest following 5 steps to find the regression model (1) using (3) and (4).

Step 1: Use the least squares method to find the intermediate estimators $\hat{l}_{A_{k}}(1)$ and $\hat{r}_{A_{k}}(1)$ of $l_{A_{k}}(1)$ and $r_{A_{k}}(1)$ by minimizing

$$
\sum_{i=1}^{n}\left(l_{Y_{i}}(1)-\sum_{k=0}^{p} l_{A_{k}}(1) \cdot l_{X_{i k}}(1)\right)^{2}=M i n !
$$

and

$$
\sum_{i=1}^{n}\left(r_{Y_{i}}(1)-\sum_{k=0}^{p} r_{A_{k}}(1) \cdot r_{X_{i k}}(1)\right)^{2}=\operatorname{Min} !
$$

respectively.

Step 2: For some $\alpha^{*} \in(0,1)$, find the intermediate estimators $\bar{l}_{A_{k}}\left(\alpha^{*}\right)$ and $\bar{r}_{A_{k}}\left(\alpha^{*}\right)$, using the formula defined in step 1. That is,

$$
\sum_{i=1}^{n}\left(l_{Y_{i}}\left(\alpha^{*}\right)-\sum_{k=0}^{p} l_{A_{k}}\left(\alpha^{*}\right) \cdot l_{X_{i k}}\left(\alpha^{*}\right)\right)^{2}=M i n !
$$

and

$$
\sum_{i=1}^{n}\left(r_{Y_{i}}\left(\alpha^{*}\right)-\sum_{k=0}^{p} r_{A_{k}}\left(\alpha^{*}\right) \cdot r_{X_{i k}}\left(\alpha^{*}\right)\right)^{2}=\operatorname{Min!}
$$

respectively. Then find the estimators $\hat{l}_{A_{k}}\left(\alpha^{*}\right)$ and $\hat{r}_{A_{k}}\left(\alpha^{*}\right)$ of $l_{A_{k}}(\alpha)$ and $r_{A_{k}}(\alpha)$ satisfying

$$
\hat{l}_{A_{k}}\left(\alpha^{*}\right)=\operatorname{Min}\left\{\bar{l}_{A_{k}}\left(\alpha^{*}\right), \hat{l}_{A_{k}}(1)\right\}
$$


and

$$
\hat{r}_{A_{k}}\left(\alpha^{*}\right)=\operatorname{Max}\left\{\bar{r}_{A_{k}}\left(\alpha^{*}\right), \hat{r}_{A_{k}}\left(\alpha^{*}\right)\right\}
$$

to be TrFNs.

Step 3: For arbitrary $\alpha^{*} \neq \alpha \in(0,1)$, find the intermediate estimators $\bar{l}_{A_{k}}(\alpha)$ and $\bar{r}_{A_{k}}(\alpha)$, using the formula defined in step 2. Then find the estimators $\hat{l}_{A_{k}}(\alpha)$ and $\hat{r}_{A_{k}}(\alpha)$ of $l_{A_{k}}(\alpha)$ and $r_{A_{k}}(\alpha)$ satisfying

$$
\hat{l}_{A_{k}}(\alpha)= \begin{cases}\operatorname{Max}\left\{\hat{l}_{A_{k}}\left(\alpha^{*}\right), \operatorname{Min}\left\{\bar{l}_{A_{k}}(\alpha), \hat{l}_{A_{k}}(1)\right\}\right\} \\ \operatorname{Min}\left\{\bar{l}_{A_{k}}(\alpha), \hat{l}_{A_{k}}\left(\alpha^{*}\right)\right\} & \text { if } \alpha^{*}<\alpha \\ & \text { if } \alpha^{*} \geq \alpha\end{cases}
$$

to be TrFNs. The $\left\{\hat{l}_{A_{k}}\left(\alpha_{j}\right): j=1, \cdots, s\right\}$ and $\left\{\hat{r}_{A_{k}}\left(\alpha_{j}\right)\right.$ : $j=1, \cdots, s\}$ estimated in step 3 are used to estimate the membership function of the regression coefficients. Note that when the membership function is given as a nonlinear curve, more number of $\alpha_{j}$ s are needed to estimate the regression coefficients.

Step 4: To estimate the membership function $M_{\hat{A}_{k}}(\cdot)$ of $\hat{A}_{k}$, we use $\alpha$-level set $\hat{A}_{k}\left(\alpha_{j}\right)=\left[\hat{l}_{A_{k}}\left(\alpha_{j}\right), \hat{r}_{A_{k}}\left(\alpha_{j}\right)\right](j=$ $1, \cdots, s)$, where $s$ is the number of $\alpha$-level sets that we are going to estimate. For this, we apply the least squares method to $\left\{\left(\hat{r}_{A_{k}}\left(\alpha_{j}\right), \alpha_{j}\right) \mid j=1, \cdots, s\right\}$ and $\left\{\left(\hat{l}_{A_{k}}\left(\alpha_{j}\right), \alpha_{j}\right) \mid j=1, \cdots, s\right\}$. We estimate the membership function $M_{\hat{A}_{k}}(\cdot)$ of $\hat{A}_{k}$ satisfying

$$
M_{\hat{A}_{k}}\left(\hat{l}_{A_{k}}(1)\right)=M_{\hat{A}_{k}}\left(\hat{r}_{A_{k}}(1)\right)=1
$$

Step 5: We find the estimated value $\hat{Y}_{i}^{1}\left(X_{i}\right)$ of $Y\left(X_{i}\right)$ using $\hat{A}_{k}(k=0, \cdots, p)$ obtained from above 4 steps and the fuzzy regression model (1) as follows:

$$
\hat{Y}_{i}^{1}\left(X_{i}\right)=\hat{A}_{0} \oplus \hat{A}_{1} \otimes X_{i 1} \oplus \cdots \oplus \hat{A}_{p} \otimes X_{i p}
$$

In ordinary regression, there is only one method to find the estimated value of responsible variable. That is, after finding the estimates of the regression coefficients we use the regression model by substitution. On the other hand, we consider two different methods in this paper to find the estimated value of $Y\left(X_{i}\right)$ of fuzzy regression model. The first method to find $\hat{Y}_{i}^{1}\left(X_{i}\right)$ after estimating $\hat{A}_{k}$ and applying substitution to fuzzy regression model following above 5 steps. The second method

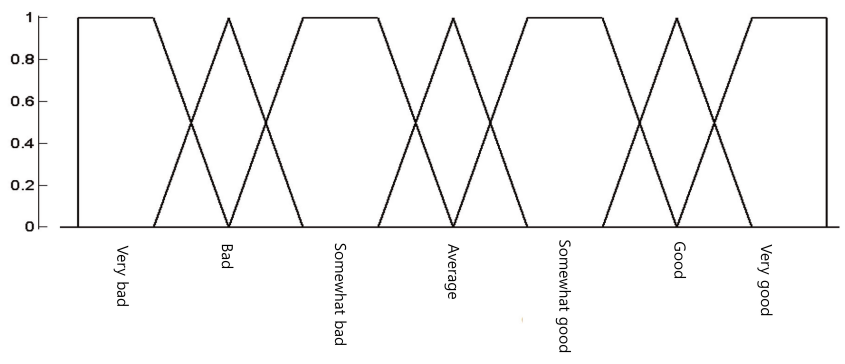

Figure 2. Fuzzy numbers for the value of real estate.

is applying above steps directly to $Y\left(X_{i}\right)$ not estimating coefficient of regression $\hat{A}_{k}$, which will be denoted by $\hat{Y}_{i}^{2}\left(X_{i}\right)$. Note that there are some methods which estimate response variables directly without estimating the regression coefficients. It might be simple to estimate $Y_{i}^{2}\left(X_{i}\right)$ directly, but this method has a drawback, which makes it unable to estimate $Y_{i}^{2}\left(X_{i}\right)$ when a new value of independent variable is given. We compare $\hat{Y}_{i}^{1}\left(X_{i}\right)$ and $\hat{Y}_{i}^{2}\left(X_{i}\right)$ through re-auction data provided in next section.

\section{Fuzzy Regression Model for Re-auction Data}

To find the statistical model for real estate re-auction data, we investigated the potential variables which may affect the number of bidders. Consequently, we found out that affect the number of total bidders has to do with the ratio between the price of lowest bid, the total number of bids, and the perceived market value of the real estate. In order to find the statistical model for the number of bidders based on the study in advance, we've actually used 18 samples of specific area of Seoul in 2013. The number of bidders on re-auction is known to be dependent on the number of bidders who participated on the original auction prior to failure of bidding. According to the expert view, it is empirically known that the number of bidders of re-auction shows specific patterns when the number of bidders of prior auction was 13, 420, and over 20. Auction participants of targeted real estate are heavily influenced by the subtle changes in the market climate [2]. Therefore, the number of bidders should reflect facts both subjective and objective, thus should be expressed in terms of trapezoidal fuzzy numbers. In addition, it is also known that the price of lowest bid of re-auction gets $20 \%$ smaller than prior auction [3]. The number of bidders just prior to the re-auction serves as the crucial factors which decides outcome. However, the main reason for failure of bidding has to do with unreasonably high winning price, so the reasonable bidding price is really important.

The real estate value at re-auction is determined by factors 
Table 1. Fuzzy data for sales volumes

\begin{tabular}{ccccc}
\hline No. & $x_{i 1}$ & $x_{i 2}$ & $X_{i 3}$ & $Y_{i}$ \\
\hline 1 & 0.8 & 2 & $(8,9,10,10)$ & $(14,16,18,20)$ \\
2 & 0.8 & 2 & $(4,5,5,6)$ & $(2,4,6,8)$ \\
3 & 0.8 & 1 & $(4,5,5,6)$ & $(0,1,2,3)$ \\
4 & 0.64 & 1 & $(8,9,10,10)$ & $(10,13,16,19)$ \\
\hline 5 & 0.64 & 1 & $(5,6,7,8)$ & $(0,1,2,3)$ \\
6 & 1 & 1 & $(2,3,4,5)$ & $(1,2,3,4)$ \\
\hline 7 & 1 & 3 & $(5,6,7,8)$ & $(1,3,6,8)$ \\
8 & 0.64 & 2 & $(5,6,7,8)$ & $(0,1,3,4)$ \\
\hline 9 & 0.8 & 4 & $(8,9,10,10)$ & $(2,4,6,8)$ \\
10 & 1 & 7 & $(4,5,5,6)$ & $(1,2,3,4)$ \\
\hline 11 & 0.8 & 2 & $(8,9,10,10)$ & $(1,2,6,7)$ \\
12 & 0.8 & 1 & $(5,6,7,8)$ & $(0,1,3,4)$ \\
\hline 13 & 0.64 & 1 & $(7,8,8,9)$ & $(2,4,6,8)$ \\
14 & 1 & 18 & $(8,9,9,10)$ & $(6,8,10,12)$ \\
\hline 15 & 1 & 10 & $(8,9,9,10)$ & $(4,6,8,10)$ \\
\hline 16 & 0.64 & 1 & $(8,9,9,10)$ & $(3,4,6,7)$ \\
\hline 17 & 0.64 & 1 & $(2,3,4,5)$ & $(1,2,3,4)$ \\
\hline 18 & 1 & 5 & $(4,5,5,6)$ & $(2,3,4,5)$ \\
\hline
\end{tabular}

such as the average lowest price of sales, location, market climate, similar sales in the neighbor, and various subjective opinions held by the auction participants. Therefore, the value of real estate at re-auction can be expressed in term of trapezoidal fuzzy numbers as follows (See Figure 2):

$(8,9,10,10)$ (very good), $(7,8,8,9)$ (good), $(5,6,7,8)$ (somewhat good), (4, 5, 5, 6)(average), $(2,3,4,5)$ (somewhat bad), $(1,2,2,3)$ (bad), $(0,0,1,2)$ (very bad).

The number of failure of bidding at re-auction can be expressed in terms of the ratio of the lowest bidding price due to failure of bidding, because it is known that the price of lowest bid of re-auction gets $20 \%$ smaller than prior auction. The fuzzy regression model for ratio of the lowest bidding price due to failure of bidding $\left(x_{i 1}\right)$, the number of bidders of prior auction $\left(x_{i 2}\right)$, investment value of the real estimate $\left(X_{i 3}\right)$, number of bid participants $Y_{i}$ is as follows:

$$
Y_{i}=A_{0} \oplus A_{1} \otimes x_{i 1} \oplus A_{2} \otimes x_{i 2} \oplus A_{3} \otimes X_{i 3} \oplus E_{i}
$$

where $Y_{i}, X_{i 3}$, and the regression coefficient $A_{j}(j=0, \cdots, 3)$ are $\operatorname{TrFN}$, and $x_{i k}(k=1,2)$ are crisp numbers $(i=1, \cdots, 18)$ (See Table 1).

The fuzzy regression model using 5 step method that we

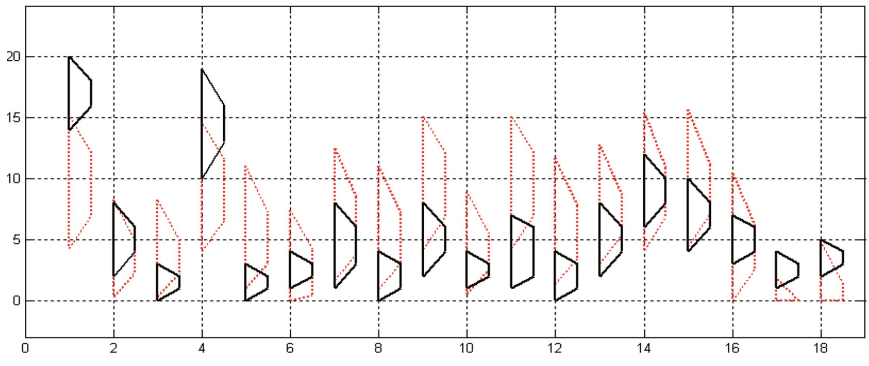

Figure 3. The observed and estimated number of bid participants: $\hat{Y}_{i}^{1}\left(X_{i}\right)$.

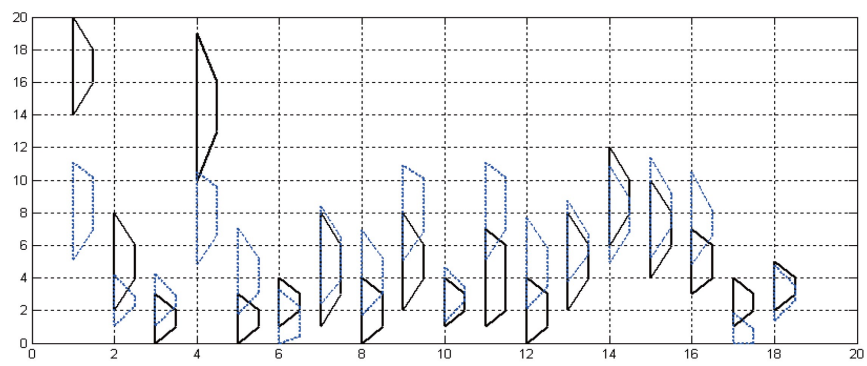

Figure 4. The observed and estimated number of bid participants: $\hat{Y}_{i}^{2}\left(X_{i}\right)$.

proposed in Section 2 for 18 samples of specific area of Seoul is as follows:

$$
\begin{aligned}
\hat{Y}_{i}^{1}\left(X_{i}\right)= & -5.34 \oplus(2.07,2.33,3.75,4.11) \otimes x_{i 1} \\
& \ominus 0.03 \otimes x_{i 2} \oplus(1.01,1.17,1.45,1.72) \otimes X_{i 3} .
\end{aligned}
$$

Here, the constant term $A_{0}$ and the coefficient $A_{2}$ of the number of bid participants of first auction $\left(x_{i 2}\right)$ are calculated as crisp numbers after applying Min-Max operation in step 3 in Section 2. Here, the 0 spreads of $\hat{A}_{0}$ and $\hat{A}_{2}$, estimated from step 1 to step 5, explains that the number of bidders of prior auction can affect only the mode of number of bid participants.

Figure 3 shows the observed number of bidders and estimated number of bidders $\hat{Y}_{i}^{1}\left(X_{i}\right)$ obtained by estimating the coefficients $A_{k}$. Figure 4 shows the observed number of bidders and estimated number of bidders $\hat{Y}_{i}^{2}\left(X_{i}\right)$ which is obtained directly from the proposed steps.

In order to compare the efficiencies of the estimated fuzzy regression model, we calculate the distance between the observed value and estimated value to find the errors for accuracies. For this, we propose distance between two fuzzy numbers as follows [2, 13, 21, 27]:

$$
M=\frac{1}{n} \sum_{i=1}^{n} d\left(Y_{i}, \hat{Y}_{i}\right),
$$


where

$$
d\left(Y_{i}, \hat{Y}_{i}\right)=\frac{\int_{-\infty}^{\infty}\left|\mu_{Y_{i}}(x)-\mu_{\hat{Y}_{i}}(x)\right| d x}{\int_{-\infty}^{\infty}\left|\mu_{Y_{i}}(x)\right| d x}+h_{d}\left(Y_{i}(0), \hat{Y}_{i}(0)\right)
$$

Here, $Y_{i}(0)$ and $\hat{Y}_{i}(0)$ are the supports of $Y_{i}$ and $\hat{Y}_{i}$. And $h_{d}$ is the Hausdorff metric defined by $h_{d}(A, B)=i n f_{a \in A} i n f_{b \in B}|a-b|$.

Table 2 shows the results of estimated values and errors obtained from (4) based on Hasdorff metric formula. It is shown in Table 2 that total sum of the error between the observed value and the estimated value $\hat{Y}_{i}^{2}$ obtained directly is more than $\hat{Y}_{i}^{1}$ which is obtained after estimating the coefficients $A_{k}$.

The estimated fuzzy regression model shows that the higher the value of real estate is, the more the number of bidders of re-auction increase. Further the larger the number of failure of bidding of auction for some real estimate is, the more the number of bidders of re-auction increase. In addition, the less the number of bid participants of first auction is, the number of bidders of re-auction increase. This result doesn't seem to coincide with the results of common re-auction. However, it seems that the increase of investment value based on the decrease of real estate market, and several times of defaults affected the results.

\section{Conclusions}

In order to find the fuzzy regression model for re-auction, we expressed the independent and dependent values in terms of trapezoidal fuzzy numbers. Eighteen data of re-auction which are collected from specific area of Seoul are used to find the fuzzy regression model. Several independent data such as ratio of the lowest bidding price due to failure of bidding, the number of bidders of prior auction, investment value of the real estimate are used to estimate the number of bid participants of re-auction. In this paper, two different least squares estimation methods are applied to fuzzy regression model. That is, estimating method applying substitution after obtaining the estimators of regression coefficients, and the other method is to estimate directly from the estimating procedure without substitution. These methods are provided in application for re-auction data, and proper performance measure is also provided to compare the accuracies. The results showed that the estimated values which are obtained directly performed better than the other method. This research shows that the number of defaults after first auction, the number of bid participants in first auction and the investment value of the real estate can affect the number of bid participants of re-auction. The number of defaults after first auction and the increase investment value can positively affect the number of bid participants of re-auction. However, the number of bid participants in first auction can negatively affect the number of bid participants of re-auction. Further research is needed to apply various methods to analyze re-auction data. And data collection covering wide scope is also needed to be considered in our next research.

\section{Conflict of Interest}

No potential conflict of interest relevant to this article was reported.

\section{References}

[1] P. Klemperer, The Economic Theory of Auctions. Cheltenham, UK: Edward Elgar, 2000.

[2] K. H. Lee, Court Practice Real Estate Auctions. Seoul, Korea: GoodAuction, 2007.

[3] H. J. Yoon, Real Estate Law. Seoul, Korea: Hakmun publishing, 2004.

[4] P. Klemperer, "Auction theory: a guide to the literature," Journal of Economic Surveys, vol. 13, no. 3, pp. 227-286, 1999. http://dx.doi.org/10.1111/1467-6419.00083

[5] L. A. Zadeh, "Fuzzy sets," Information and Control, vol. 8, no. 3, pp. 338-353, 1965. http://dx.doi.org/10.1016/S00199958(65)90241-X

[6] L. A. Zadeh, "The concept of a linguistic variable and its application to approximate reasoning I," Information Sciences, vol. 8, no. 3, pp. 199-249, 1975. http://dx.doi. org/10.1016/0020-0255(75)90036-5

[7] H. Tanaka and I. Hayashi, "Possibilistic linear regression analysis for fuzzy data," European Journal of Operational Research, vol. 40, no. 3, pp. 389-396, 1989. http://dx.doi. org/10.1016/0377-2217(89)90431-1

[8] H. Tanaka, S. Uejima, and K. Asai, "Linear regression analysis with fuzzy model," IEEE Transactions on Systems, Man, and Cybernetics, vol. 12, no. 6, pp. 903-907, 1982. http://dx.doi.org/10.1109/TSMC.1982.4308925

[9] A. R. Arabpour and M. Tata, "Estimating the parameters of a fuzzy linear regression model," Iranian Journal of Fuzzy Systems, vol. 5, no. 2, pp. 1-19, 2008. 
Table 2. Estimated values $\hat{Y}_{i}^{1}$ and $\hat{Y}_{i}^{2}$ and errors

\begin{tabular}{ccccc}
\hline No. & $\hat{Y}_{i}^{1}$ & Error & $\hat{Y}_{i}^{2}$ & Error \\
\hline 1 & $(4.31,6.96,12.12,15.13)$ & 2.2182 & $(5.10,6.96,10.13,11.06)$ & 2.7347 \\
\hline 2 & $(0.27,2.29,4.86,8.23)$ & 0.2380 & $(1.07,2.29,2.86,4.16)$ & 2.1868 \\
\hline 3 & $(0.31,2.32,4.89,8.26)$ & 2.1442 & $(1.11,2.32,2.89,4.23)$ & 2.1380 \\
4 & $(4.01,6.62,11.56,14.50)$ & 2.1820 & $(4.81,6.62,9.56,10.47)$ & 2.0685 \\
\hline 5 & $(0.99,3.12,7.20,11.05)$ & 2.3592 & $(1.78,3.12,5.20,7.02)$ & 2.2911 \\
\hline 6 & $(0,0.46,4.19,7.36)$ & 1.7725 & $(0,0.46,2.19,3.32)$ & 1.9045 \\
\hline 7 & $(1.66,3.89,8.48,12.47)$ & 1.5775 & $(2.45,3.89,6.48,8.37)$ & 1.5775 \\
8 & $(0.95,3.08,7.16,11.02)$ & 2.0267 & $(1.75,3.08,5.16,6.95)$ & 2.0265 \\
\hline 9 & $(4.23,6.89,12.06,15.06)$ & 2.1795 & $(5.03,6.89,10.06,10.93)$ & 2.1708 \\
\hline 10 & $(0.50,2.58,5.44,8.88)$ & 0.1570 & $(1.30,2.58,3.43,4.67)$ & 1.7917 \\
\hline 11 & $(4.31,6.96,12.12,15.13)$ & 2.1411 & $(5.10,6.96,10.13,11.06)$ & 2.1271 \\
\hline 12 & $(1.32,3.49,7.80,11.71)$ & 2.1381 & $(2.11,3.49,5.80,7.68)$ & 2.1296 \\
\hline 13 & $(3.00,5.45,8.65,12.78)$ & 1.8626 & $(3.80,5.45,6.65,8.74)$ & 1.8626 \\
\hline 14 & $(4.13,6.87,10.87,15.40)$ & 0.9088 & $(4.93,6.87,8.86,10.86)$ & 1.7841 \\
\hline 15 & $(4.42,7.15,11.15,15.67)$ & 1.7869 & $(5.22,7.15,9.14,11.37)$ & 1.7869 \\
\hline 16 & $(4.01,6.62,10.11,14.50)$ & 2.1705 & $(4.81,6.62,8.11,10.47)$ & 2.1605 \\
\hline 17 & $(0,0,2.84,5.88)$ & 0.0064 & $(0,0,0.84,1.84)$ & 2.2442 \\
\hline 18 & $(0.57,2.65,5.51,8.95)$ & 2.6700 & $(1.37,2.65,3.50,4.79)$ & 1.7477 \\
\hline$M$ & & 1.6966 & & 2.0407 \\
\hline
\end{tabular}

[10] A. Bisserier, R. Boukezzoula, and S. Galichet, "A revisited approach to linear fuzzy regression using trapezoidal fuzzy intervals," Information Sciences, vol. 180, no. 19, pp. 3653-3673, 2010. http://dx.doi.org/10.1016/j.ins.2010. 06.017

[11] A. Bisserier, R. Boukezzoula, and S. Galichet, "Linear fuzzy regression using trapezoidal fuzzy intervals," Journal of Uncertain Systems, vol. 4, no.1, pp. 59-72, 2010.

[12] C. H. Cheng, J. R. Chang, and C. A. Yeh, "Entropy-based and trapezoid fuzzification-based fuzzy time series approaches for forecasting IT project cost," Technological Forecasting and Social Change, vol. 73, no. 5, pp. 524542, 2006. http://dx.doi.org/10.1016/j.techfore.2005.07. 004

[13] H. Y. Jung, J. H. Yoon, and S. H. Choi, "Fuzzy linear regression using rank transform method," Fuzzy Sets and Systems, vol. 274, pp. 97-108, 2015. http://dx.doi.org/10. 1016/j.fss.2014.11.004

[14] H. T. Liu, "An improved fuzzy time series forecasting method using trapezoidal fuzzy numbers," Fuzzy Opti- mization and Decision Making, vol. 6, no. 1, pp. 63-80, 2007. http://dx.doi.org/10.1007/s10700-006-0025-9

[15] A. Maleki, E. Pasha, and T. Razzaghnia, "Possibility linear regression analysis with trapezoidal fuzzy data," World Applied Sciences Journal, vol. 18, no. 1, pp. 37-42, 2012.

[16] H. Y. Jung, W. J. Lee, and J. H. Yoon, "A unified approach to asymptotic behaviors for the autoregressive model with fuzzy data," Information Sciences, vol. 257, pp. 127-137, 2014. http://dx.doi.org/10.1016/j.ins.2013.09.024

[17] H. K. Kim, J. H. Yoon, and Y. Li, "Asymptotic properties of least squares estimation with fuzzy observations," Information Sciences, vol. 178, no. 2, pp. 439-451, 2008. http://dx.doi.org/10.1016/j.ins.2007.07.010

[18] W. J. Lee, H. Y. Jung, J. H. Yoon, and S. H. Choi, "The statistical inferences of fuzzy regression based on bootstrap techniques," Soft Computing, vol. 19, no. 4, pp. 883-890, 2015. http://dx.doi.org/10.1007/s00500-014-1415-5

[19] R. E. Giachetti and R. E. Young, "A parametric representation of fuzzy numbers and their arithmetic operators," 
Fuzzy Sets and Systems, vol. 91, no. 2, pp. 185-202, 1997. http://dx.doi.org/10.1016/S0165-0114(97)00140-1

[20] R. R. Yager, "Using trapezoids for representing granular objects: applications to learning and OWA aggregation," Information Sciences, vol. 178, no. 2, 363-380, 2008. http: //dx.doi.org/10.1016/j.ins.2007.08.015

[21] S. H. Choi and J. J. Buckley, "Fuzzy regression using least absolute deviation estimators," Soft Computing, vol. 12, no. 3, pp. 257-263, 2007. http://dx.doi.org/10.1007/ s00500-007-0198-3

[22] A. Kumar, J. Kaur, and P. Singh, "Solving fully fuzzy linear programming problems with inequality constraints," International Journal of Physical and Mathematical Sciences, vol. 1, pp. 6-17, 2010.

[23] S. H. Nasseri, E. Behmanesh, F. Taleshian, M. Abdolalipoor, and N. A. Taghi-Nezhad, "Fully fuzzy linear programming with inequality constraints," International Journal of Industrial Mathematics, vol. 5, no. 4, pp. 309316, 2013.

[24] J. H. Yoon and S. H. Choi, "Fuzzy least squares estimation with new fuzzy operations," Advances in Intelligent Systems and Computing, vol. 190, pp. 193-202, 2013. http://dx.doi.org/10.1007/978-3-642-33042-1_21

[25] A. K. Shaw and T. K. Roy, "Generalized trapezoidal fuzzy number with its arithmetic operations and its application in fuzzy system reliability analysis," International Journal of Pure and Applied Sciences and Technology, vol. 5, no. 2, pp. 60-76, 2011.
[26] M. S. Sin and N. H. Cho, "Fault tree analysis model based on trapezoidal fuzzy number," Journal of the Korean Society for Quality Management, vol. 20, no. 1, pp. 118-125, 1992.

[27] M. Namdari, J. H. Yoon, A. Abadi, S. M. Taheri, and S. H. Choi, "Fuzzy logistic regression with least absolute deviations estimators," Soft Computing, vol. 19, no. 4, pp. 909-917, 2015. http://dx.doi.org/10.1007/s00500-0141418-2

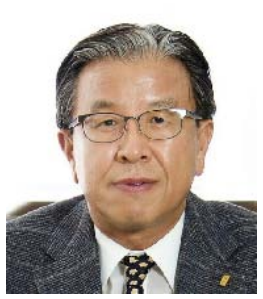

Il Kyu Kim obtained his Ph.D. degree in Mathematics majoring mathematica statistics from Yonsei University, Seoul, Korea in 1998. He is currently full professor of Gwanju University. His main research interests are mathematical prediction method using the soft computing and statistical prediction in real estate investment and theory of real estate auction.

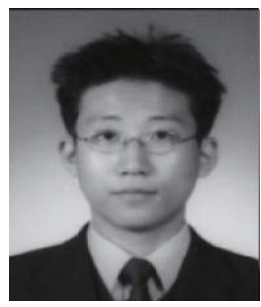

Woo-Joo Lee obtained a Ph.D. in Mathematics majoring mathematical statistics from Yonsei University, Seoul, Korea. His main research interests are data mining, fuzzy time series and intelligent systems. 


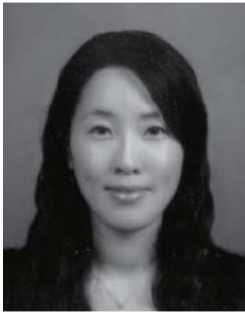

Jin Hee Yoon received Bachelor, Master and Ph.D. degree in Mathematics from Yonsei university. She is currently faculty of school of Mathematics and Statistics at Sejong University, Seoul, Korea. Her research interests are fuzzy regression analysis, fuzzy time series and F-transform. She is a board member of KIIS (Korean institute of Intelligent Systems) and has been working as an editor, guest editor and editorial board member of The Scientific world journal, Information, International journal of Applied Economics and International Journal of Fuzzy logic and Intelligent Systems etc. Also, she has been working as an organizer and committee member of several international conferences such as 2012, 2013
NIMS Hot topic workshop, 2012, 2013 ICIS, 2014, 2015 FUZZ IEEE, 2015 ISIS, 2016 SCIS\&ISIS, 2016 ICFMsquare 2016, ICMF 2016 etc.

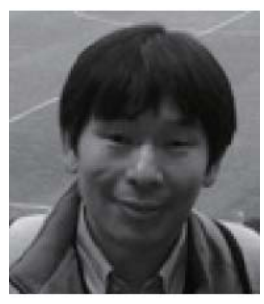

Seung Hoe Choi obtained his Ph.D. degree in Mathematical Statistics from Yonsei University Korea in 1994. Since 1996, he is currently full professor of Korea Aerospace University. His main research interests are mathematical prediction method using the soft computing and statistical prediction in sports like soccer, baseball and basketball. 